ISSN: 2174-5609

DOI. https://doi.org/10.14198/INTURI2022.23.6

†Tा Investigaciones

$\triangle$ S TURISTICAS

Cita bibliográfica: Alberca-Sialer, F.A.y Soto-Cáceda, D.R. (2022). Patrimonio cultural inmaterial y turismo. Análisis de la producción científica publicada en revistas iberoamericanas de turismo. Investigaciones Turísticas (23), pp.112-139. https://doi.org/10.14198/INTURI2022.23.6

\title{
Patrimonio cultural inmaterial y turismo. Análisis de la producción científica publicada en revistas iberoamericanas de turismo
}

Intangible cultural heritage and tourism. Analysis of scientific production published in IberoAmerican tourism journals

Fabrizio Augusto Alberca-Sialer (iD), Universidad de San Martin de Porres, Perú fabrizioalberca@gmail.com

Delia Rosa Soto-Cáceda (iD, Pontificia Universidad Católica de Perú, Perú deliasc@gmail.com

\section{RESUMEN}

El turismo basado en el patrimonio cultural inmaterial (PCI) viene ganando protagonismo dentro de las prácticas turísticas actuales, al igual que en los estudios turísticos. En ese sentido, el presente estudio bibliométrico se plantea caracterizar la producción y publicaciones en revistas de turismo iberoamericanas para conocer los avances y vacíos de la investigación sobre $\mathrm{PCl}$. Se analizaron 59 artículos procedentes de 12 revistas de turismo. Se identificó que, pese a que el turismo cultural patrimonial es tendencia mundial, las publicaciones aquí analizadas no lo reflejan. Las publicaciones regionales hacen honor a su ámbito geográfico tanto en la procedencia de sus autores como en los temas tratados, lo que da a este trabajo una mirada netamente regional. No existe una tendencia marcada de publicaciones ni de autores que se dediquen a la investigación y publicación sistemáticas sobre $\mathrm{PCl}$ en su relación con el turismo. Brasil y España sobresalen como los países más prolíficos en cuanto a cantidad de autores e instituciones afiliadoras, y sobresalen también los temas relacionados al uso turístico del patrimonio, la educación patrimonial y los impactos del turismo. Estos resultados permiten a investigadores en turismo o $\mathrm{PCl}$ ubicar artículos especializados sobre lberoamérica y a editores de las revistas regionales de turismo reconocer la carencia de artículos sobre $\mathrm{PCl}$, así como la necesidad de publicar en inglés para ganar visibilidad. En adelante, las investigaciones deberán incorporar la recuperación poscovid-19 dentro de un enfoque sostenible del uso turístico del PCl.

Palabras clave: Bibliometría; patrimonio cultural inmaterial; turismo; turismo cultural. 


\section{ABSTRACT}

Tourism based on intangible cultural heritage $(\mathrm{ICH})$ has been gaining prominence within current tourism practices, and also in tourism studies. In this sense, this bibliometric study aims to characterize the production and publications in Ibero-American tourism journals in order to determine the advances and gaps in research on ICH. Fifty-nine articles from 12 tourism journals were analyzed. The findings reveal that, although cultural heritage tourism is a global trend, the publications analyzed here do not reflect this. Regional publications honour their geographical scope both in the origin of their authors and in the topics covered, which gives this work a purely regional perspective. There is no marked tendency for publications or authors to engage in systematic research and publication on $\mathrm{ICH}$ in its relationship with tourism. Brazil and Spain stand out as the most prolific countries in terms of the number of authors and affiliate institutions, and issues related to the tourist use of heritage, heritage education and the impacts of tourism are also prominent. These results allow researchers in tourism or ICH to locate specialized articles on Ibero-America and editors of regional tourism journals to recognize the lack of articles on $\mathrm{ICH}$, as well as the need to publish in English to gain visibility. Future research should incorporate post-COVID-19 recovery within a sustainable approach to tourism use of the $\mathrm{ICH}$.

Keywords: Bibliometrics; intangible cultural heritage; tourism; cultural tourism.

\section{I.INTRODUCCIÓN}

El patrimonio cultural es un conjunto de expresiones tangibles e intangibles en las que una comunidad se autoreconoce y se hace reconocible. Es el legado del pasado, que vive en el presente y debe transmitirse a futuras generaciones (UNESCO, 2008), fungiendo como medio de comunicación intergeneracional.

De este modo, el patrimonio cultural, en sus manifestaciones tangible e intangible, es parte de la oferta turística (World Tourism Organization, 2018). Y es que el turismo a nivel mundial es, en un importante porcentaje, de tipo cultural propiamente dicho o así identificable: $35,8 \%$; incluso es en muchos casos el atractivo principal el patrimonio cultural tangible e intangible (World Tourism Organization, 2018).

En particular, el patrimonio cultural inmaterial ha venido ganando importancia tanto en el turismo (Espeso-Molinero, 2019; Olivera, 2011; Richards, 2018a, 2018b; Ross et al., 2017; World Tourism Organization, 2020) como en las industrias creativas (Cominelli \& Greffe, 2012; Espeso-Molinero, 2019; Richards, 2018b, 2018a). Queda claro entonces que el patrimonio puede ser considerado un recurso - no renovable - (Álvarez-García, Maldonado-Erazo, Del Río-Rama, \& Castellano-Álvarez, 2019; Hernández, 2002) y el turismo ser uno de sus usos sociales, además de promotor del desarrollo local (Álvarez-García, Maldonado-Erazo, Del RíoRama, \& Castellano-Álvarez, 2019; Garcia Canclini, 1999; Roigé et al., 2019).

En los últimos años, el turismo se ha venido diversificando, motivo por el cual ha buscado nuevos públicos. En ese sentido, el patrimonio cultural inmaterial ( $\mathrm{PCl}$ ), en particular, ha emergido como un elemento valioso e importante, ya que a través suyo los turistas pueden encontrar autenticidad y cercanía con los visitados; los visitantes, consiguientemente, encuentran valor añadido en él (Espeso-Molinero, 2019; Roigé et al., 2019).

El turismo como disciplina científica se encuentra aún en desarrollo (Mulet-Forteza et al., 2019; Muñoz de Escalona, 2017), aunque es posible también que se encuentre ya en sus 
etapas iniciales de maduración (Timothy, 2018). No obstante, en líneas generales, el estudio sistemático, organizado y riguroso de la producción científica (es decir, un estudio bibliométrico) se presenta como oportunidad y necesidad para evaluar el avance disciplinar.

A propósito, un signo de su desarrollo inicial o maduración tiene que ver con la aparición y proliferación de revistas especializadas en turismo, lo cual es reflejo del creciente interés de la academia y las sociedades por entender el tema, algo previsible dado los impactos económico, social, cultural y ambiental que el turismo genera en el mundo entero, en un contexto de continuo crecimiento ${ }^{1}$ (Organización Mundial del Turismo, 2019).

La investigación sobre el turismo basado en el patrimonio cultural empieza a tomar protagonismo desde la década de 1980, acelerando su desarrollo en la de 1990, aunque a través de estudios de carácter descriptivo (Timothy, 2018). Es, en realidad, con el nuevo milenio que empiezan los esfuerzos por entender el tema de manera más profunda, con claro énfasis en la dimensión experiencial entre la gente y el lugar de visita y en las amplias implicancias sociales del patrimonio de uso turístico (Timothy, 2018). Además, es en estos tiempos que el patrimonio cultural empieza a ganar espacio como parte de la oferta turística (Roigé et al., 2019). No obstante, el patrimonio cultural como objeto de estudio es complejo y multifacético, y recién se está empezando a entender dentro de los estudios del área de turismo (Timothy, 2018). En cuanto a la relación entre el PCl y el turismo, es ambivalente, importante y de retroalimentación (Roigé et al., 2019), además de, a la luz de lo dicho, aún poco estudiada.

Por otro lado, la bibliometría es una herramienta útil para el análisis de la evolución y progreso de las disciplinas (Koseoglu et al., 2016; Okumus et al., 2018; Quevedo-Silva et al., 2016; Župič y Čater, 2015); es un estudio de tipo cuantitativo que permite evaluar objetivamente la producción investigativa en un determinado campo y periodo de tiempo (Figueroa-Domecq et al., 2015; Župič y Čater, 2015), a través de la clasificación de datos de estudios publicados, incluyendo por ejemplo, citas, afiliaciones institucionales, palabras clave, temas discutidos, métodos empleados. Se basa además en el uso de técnicas estadísticas básicas o avanzadas (Koseoglu et al., 2016).

En los estudios bibliométricos pueden distinguirse dos métodos: uno básico, que contiene métricas de los artículos y autores, y otro que incluye análisis de co-ocurrencia (como cocitación, coautoría y copalabras) (Koseoglu, Sehitoglu y Craft, 2015, como se citó en Koseoglu et al., 2016). Los estudios bibliométricos, así también, se clasifican en descriptivos, que incorporan a los que en líneas generales presentan los temas más estudiados, grupos de investigación, revistas que más publican, principales autores y métodos utilizados. En tanto que las otras clasificaciones se enfocan o bien en los aspectos metodológicos o bien en el análisis cuantitativo con el fin de presentar tendencias de investigación y propuestas teóricas para el desarrollo de cada tema (Quevedo-Silva et al., 2016). La presente investigación, por cierto, es claramente un estudio descriptivo que incluye métricas de artículos y análisis de coocurrencia de palabras clave.

Así, los estudios bibliométricos se categorizan en 3 grupos: estudios de revisión, estudios evaluativos y estudios relacionales (Koseoglu et al., 2016), siendo de importancia específica para la presente investigación los primeros, pues como señalan los mismos autores,

\footnotetext{
${ }^{1}$ Crecimiento que se ha visto estancado debido a la pandemia generada por la covid-19 y la incertidumbre global consecuente.
} 
generan conocimiento a través de análisis de frecuencias o estadística básica de estudios publicados; y los relacionales, que lo hacen a partir de las co-ocurrencias de palabras, autores, entre otros. Los evaluativos, en tanto, como información, lo hacen, por ejemplo, a través de medidas de la productividad o de impacto.

Respecto de los estudios bibliométricos en turismo, el primero fue realizado en 1989 por Weaver y McCleary ( Durán-Sánchez et al., 2017). Desde ahí se han venido desarrollando estudios bibliométricos sobre diferentes aspectos relacionados al turismo y, en algunos casos, en relación con el patrimonio cultural (aunque no directamente en relación con el $\mathrm{PCl}$ ), estando algunos orientados a la comida, gastronomía y turismo (Ellis et al., 2018; MaldonadoErazo et al., 2019; Okumus et al., 2018; Durán-Sánchez et al., 2017; Santucci et al., 2018), el turismo en sus aspectos económicos, teóricos y de sostenibilidad (Comerio y Strozzi, 2019; Graciano y Holanda, 2020; Koseoglu et al., 2016; Meneguel et al., 2019; Mota et al., 2018; Mulet-Forteza et al., 2019; Peña et al., 2019; Ruhanen et al., 2015), la hotelería u hospitalidad (García-Lillo et al., 2016; López-Bonilla \& López-Bonilla, 2020), el género (Figueroa-Domecq et al., 2015), lo metodológico (Koc y Boz, 2014), el turismo religioso (Durán-Sánchez et al., 2018; Mora-Torres et al., 2017), el turismo e industrias creativas (Álvarez-García et al., 2019; Galvagno y Giaccone, 2019), el patrimonio cultural (Álvarez-García et al., 2019; Su et al., 2019) y la medición de la producción académica (Corral y Cànoves, 2013; López-Bonilla et al., 2018; Peña et al., 2019; Strandberg et al., 2018).

Además, los estudios bibliométricos son relevantes para el análisis de la evolución de las diferentes disciplinas científicas (Durán-Sánchez et al., 2018; Graciano \& Holanda, 2020). En el caso particular del turismo, cabe señalar que en los últimos años se incrementaron las publicaciones sobre la especialidad (Mulet-Forteza et al., 2019), así como de estudios bibliométricos (López-Bonilla \& López-Bonilla, 2020). Estos sirven, entonces, para repasar lo avanzado e identificar los vacíos por llenar. De ahí que sintetizar las investigaciones publicadas es una de las formas de avanzar en una particular línea o área de investigación (Župič y Čater, 2015).

El patrimonio cultural, en tanto tema de primera importancia para el turismo, no se ha visto sometido a un estudio bibliométrico desde la perspectiva del turismo, motivo por el cual la presente investigación, se cree, es pertinente y oportuna. Como se ha mencionado, el turismo mundial es pues en gran medida de tipo cultural y se basa en el patrimonio (World Tourism Organization, 2018), contexto en el que el PCl viene ganando protagonismo (EspesoMolinero, 2019; Olivera, 2011; World Tourism Organization, 2020).

Por otro lado, exponer contenidos de los artículos de las revistas de turismo de Iberoamérica resulta por demás relevante, pues las investigaciones bibliométricas existentes en turismo se han centrado preferentemente en publicaciones en revistas indizadas en bases de datos más globales, excluyendo las publicaciones de bases regionales. Este vacío se pretende subsanar con la presente investigación. A propósito y como ejemplo, se calcula que el porcentaje de participación, según región geográfica, en las principales revistas de turismo -no iberoamericanas- es: Sudamérica (que concentra gran parte de países iberoamericanos; de América Central no se presenta información) en Annals of Tourism Research alcanzaba el 1,1 \% de participación, en Cornell Hotel and Restaurant Quarterly el 0,4 $\%$, en International Journal of Hospitality Managment el 1,2 \%, en Journal of Hospitality Research no presenta datos, en Journal of Travel Research el 0,6\%, en Toursim Managment el 0,6\%, es decir, la participación total de autores de procedencia sudamericana llega apenas 
al 1,2 \%, mientras que, por ejemplo, quienes proceden de Europa alcanzan al 27,1 \%, de América del Norte 41,2 \% y de Asia 22 \% (Svensson et al., 2009). En otras palabras, lo investigado en la región iberoamericana es poco visible, debido a la baja participación de los autores, de ahí la importancia de la presente investigación.

Así también, y como se verá con mayor detalle, el 96,55 \% de los artículos de la presente investigación tiene como lugar de estudio un país iberoamericano, mientras que las revistas y artículos de bases de datos de mayor alcance geográfico, o globales, reúnen y presentan realidades de diferentes partes del mundo (Svensson et al., 2009). La presente investigación permitirá, entonces, reflejar la realidad de los países iberoamericanos en cuanto a producción, publicaciones y resultados. Por ende, el objetivo será caracterizar la producción científica sobre el patrimonio cultural inmaterial publicada en artículos de revistas iberoamericanas indizadas en bases de datos de la región.

\section{II.METODOLOGÍA}

Los estudios bibliométricos son trabajos cuantitativos que pueden ser básicos o complejos (Koseoglu et al., 2016) y que permiten delinear el comportamiento de un área de conocimiento para poder avanzar en su desarrollo (Graciano y Holanda, 2020; Župič y Čater, 2015).

Los artículos científicos, por su lado "(publicados en revistas especializadas), son la forma primordial de comunicación científica" (Maletta, 2015, p. 418), por tal motivo, en esta investigación serán analizados. Su búsqueda, identificación y selección se hizo durante los primeros días del mes de noviembre del año 2019. Cabe precisar que se ha trabajado solo con artículos científicos de carácter empírico, es decir, con aquellos que presentan como mínimo metodología y resultados obtenidos tras la recolección y análisis de datos de la realidad, y que, además, fueran de acceso abierto (open access). Por ende, no se incluyeron artículos teóricos, ensayos, reseñas, otros.

El proceso de selección tuvo como primer filtro la identificación de revistas indizadas simultáneamente en las bases de datos Redalyc y Scielo. Seguidamente, a fin de tener una mirada más amplia del tema, se decidió (intencionalmente) agregar 6 importantes revistas iberoamericanas de turismo incluidas en DOAJ, WoS y/o Scopus: Turismo y Patrimonio, Turismo y Sociedad, Investigaciones Turísticas, Tourism \& Management Studies, European Journal of Tourism Hospitality and Recreation y Enlightening Tourism, esto se debe a los altos estándares de calidad a las que se encuentran sometidas. Cabe precisar que el número más antiguo data del año 1991, de la revista Estudios y Perspectivas en Turismo. Es decir, la búsqueda y revisión abarcó un marco temporal de 28 años (entre 1991 y 2019).

Las revistas finalmente identificadas para el estudio fueron: Estudios y Perspectivas en Turismo, Revista Brasileira de Pesquisa em Turismo (RBTUR), Caderno Virtual de Turismo, PASOS - Revista de Turismo y Patrimonio Cultural, Cuadernos de Turismo, El Periplo Sustentable, Gestión Turística, Podium: Sport, Leisure and Tourism Review, Turismo: Visão e Ação, a las que se les sumaron las 6 seleccionadas intencionalmente: Turismo y Patrimonio, Turismo y Sociedad, Investigaciones Turísticas, Tourism \& Management Studies, European Journal of Tourism Hospitality and Recreation y Enlightening Tourism. Es decir, en esta investigación se revisaron en total 15 revistas iberoamericanas de turismo indizadas. De estas, tras la revisión, se identificó que solo en 12 había artículos sobre patrimonio cultural 
inmaterial. Este número de revistas, por cierto, es mayor del que usualmente (9) se analizan en investigaciones bibliométricas sobre turismo (López-Bonilla et al., 2018), lo cual revela la amplitud de la investigación.

En las 12 revistas, entonces, se identificaron 330 documentos aparentemente asociados al tema patrimonio cultural inmaterial en relación con el turismo. Para ello, se utilizaron como términos de búsqueda (en castellano, portugués e inglés): patrimonio cultural inmaterial, patrimonio cultural intangible, patrimonio inmaterial y patrimonio intangible, los que debían encontrarse en el título, resumen y/o palabras clave. Estos 330 textos no eran necesariamente de naturaleza empírica porque, en esta primera revisión, se decidió incorporar a la base de datos creada a todo artículo que tratara sobre $\mathrm{PCl}$. Se decidió proceder de ese modo para evitar excluir algún texto que cumpliera con las condiciones establecidas. Posteriormente y por etapas, se fue afinando la selección de los artículos, por lo que se volvió a revisar la presencia de los términos de búsqueda establecidos en los 330 textos, verificando, ahora sí, que sean efectivamente artículos científicos de naturaleza empírica. Así, de los 330 documentos identificados inicialmente, se pasó a 97. Finalmente, la cantidad de artículos seleccionados se redujo a 59, ya que dentro de los 97 seleccionados se hallaron algunos que no tenían como tema principal el uso turístico del $\mathrm{PCl}$, pese a incluir los términos de búsqueda y ser científicos, pues podían ser la descripción de una manifestación del $\mathrm{PCl}$, o tratar sobre patrimonio tangible (y mencionar al PCl indirectamente), por citar un par de ejemplos. Cabe señalar que se incluyeron artículos en idiomas castellano, portugués e inglés.

Para el desarrollo de la investigación se siguieron 4 etapas: (1) documentación; (2) obtención, tratamiento, organización y tabulación de datos; (3) análisis de la información y; (4) elaboración y difusión del informe final con resultados obtenidos (Moreno-Fernández y Moreno-Crespo, 2016, como se citó en Peña et al., 2019). La validación de este tipo de investigaciones, en tanto, depende de la selección de bases de datos y que cubran suficientemente el tema bajo análisis (Bordons y Zulueta, 1999, como se citó en DuránSánchez et al., 2018). En ese sentido, las bases seleccionadas en la presente investigación han sido Redalyc, Scielo, DOAJ, WoS y/o Scopus. Así también, se consideraron 3 condiciones para la selección de las bases en el contexto de estudios bibliométricos: disponibilidad, relevancia y confiabilidad de la información (Rueda et al., 2007, como se citó en Álvarez-García, Maldonado-Erazo, Del Río-Rama, \& Castellano-Álvarez, 2019), cumpliéndose estas características en las seleccionadas para esta investigación.

En el presente estudio bibliométrico, entonces, se identificó la cantidad de artículos sobre $\mathrm{PCl}$ por revista y año de publicación; la cantidad de autores por artículo, así como a los autores con más artículos publicados; las filiaciones institucionales: países e instituciones según autores; también la procedencia académica de los autores (instituto, departamento, escuela, otros); las principales temáticas y enfoques metodológicos; así como los análisis de co-ocurrencia de palabras en las palabras clave.

De los 59 artículos seleccionados, se obtuvo un total 196 palabras clave asignadas por los autores; para recopilar y organizar los términos se utilizó el gestor bibliográfico Mendeley, el cual también facilitó la exportación de estos al programa VOSviewer (Van Eck \& Watman, 2010), para la visualización de densidad y las redes de co-ocurrencia. Las matrices de coocurrencia se elaboraron con la finalidad de mostrar las relaciones entre cada dos palabras clave (Gálvez, 2018) y entre aquellas con frecuencia mayor a 5: red de co-ocurrencia de dos palabras (Figura 5) y la red de co-ocurrencia de cinco palabras (Figura 6). De acuerdo a las 
figuras 5 y 6 , los conglomerados de los términos con mayor co-ocurrencia son, entre otros, "turismo", "patrimonio", "patrimonio cultural", "turismo cultural" y "patrimonio cultural inmaterial", los cuales establecen grupos de términos que se relacionan semánticamente (figura 7). Finalmente, en la figura 6 se visualiza la red de co-ocurrencia de cinco términos representando los ejes temáticos con mayor actividad.

Así, la importancia de la co-ocurrencia de palabras estriba en que ayuda a construir una estructura conceptual (Župič \& Čater, 2015). En otros términos, la aparición o repetición de palabras en documentos indican que los conceptos detrás están relacionados, y puede ser aplicado, como en este estudio, en palabras clave y resúmenes, aunque también en títulos y textos completos (Župič \& Čater, 2015).

Por último, se contrastarán los resultados con los aspectos teóricos ya presentados y, ante la ausencia de investigaciones precedentes directas, se ha creído conveniente hacer la discusión de los resultados con trabajos que vinculen las manifestaciones específicas del PCI con el turismo, como los casos de comida y gastronomía, religión, turismo comunitario basado en el patrimonio y desarrollo basado en el patrimonio.

\section{III.RESULTADOS Y DISCUSIÓN}

\subsection{Revistas seleccionadas y cantidad de artículos}

La revista iberoamericana con mayor cantidad de artículos sobre el PCl fue Pasos Revista de Turismo y Patrimonio Cultural con 19 artículos científicos (equivalentes al 32,20\%), seguida de Estudios y Perspectivas en Turismo con 9 artículos (15,25 \%). Les siguen Turismo y Patrimonio con 6 artículos científicos (10,17\%), Caderno Virtual de Turismo y RBTUR Revista Brasileira de Pesquisa em Turismo, ambas con 5 artículos (8,47 \% cada una). Cuadernos de Turismo presenta 4 artículos (6,78\%), El Periplo Sustentable y Turismo y Sociedad presentan 3 artículos (5,08 \%) cada una, Investigaciones Turísticas 2 artículos (3,39 \%) y Turismo - Visão e Ação, European Journal of Tourism Hospitality and Recreation y Tourism \& Management Studies, un artículo cada una (1,69\%).

Figura 1. Revistas iberoamericanas de turismo identificadas y cantidad de artículos

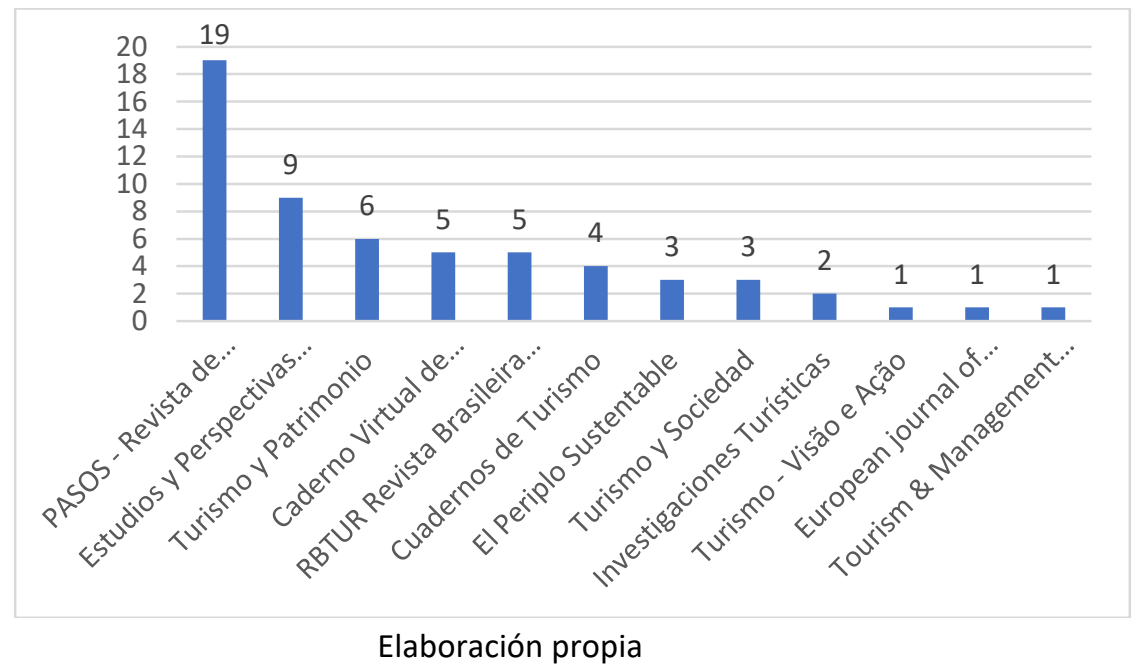


Nótese que los artículos que abordan los temas de $\mathrm{PCl}$ y turismo son relativamente pocos si se tiene en cuenta que en su conjunto equivalen al 1,20 \% del total (4910 artículos publicados y revisados en las 15 revistas). Visto así, el PCl en las publicaciones es un tema que comparativamente despierta poca atención por parte de investigadores y/o editores de las revistas, lo que llama la atención, ya que el turismo basado en el $\mathrm{PCl}$ es una de las tendencias del turismo cultural (Espeso-Molinero, 2019; Richards, 2018a; Timothy, 2018; World Tourism Organization, 2018, 2020), al igual que el turismo creativo específicamente, que se basa fundamentalmente en el PCI (Alberca, 2019; Cominelli \& Greffe, 2012; Molina, 2016; Richards, 2018b; Tan et al., 2013). Las publicaciones sobre el PCl en revistas iberoamericanas de turismo entonces parecen no ir al mismo ritmo de las tendencias, pese a que internacionalmente el turismo patrimonial domina la investigación académica (Timothy, 2018).

Respecto al idioma de publicación, 36 (61,02 \%) artículos estuvieron en castellano, 18 $(30,51 \%)$ en portugués y $5(8,47 \%)$ en inglés.

Tabla 1. Frecuencia y porcentajes de artículos según revistas iberoamericanas de turismo

\begin{tabular}{|c|c|c|c|c|}
\hline Revistas iberoamericanas & Institución editora & $\begin{array}{l}\text { País de } \\
\text { editorial }\end{array}$ & Frecuencia & Porcentaje \\
\hline $\begin{array}{c}\text { PASOS - Revista de Turismo y } \\
\text { Patrimonio Cultural }\end{array}$ & $\begin{array}{l}\text { Universidad de La } \\
\text { Laguna }\end{array}$ & España & 19 & $32,20 \%$ \\
\hline $\begin{array}{c}\text { Estudios y Perspectivas en } \\
\text { Turismo }\end{array}$ & $\begin{array}{c}\text { Centro de } \\
\text { Investigaciones y } \\
\text { Estudios Turísticos }\end{array}$ & Argentina & 9 & $15,25 \%$ \\
\hline Turismo y Patrimonio & $\begin{array}{l}\text { Universidad de San } \\
\text { Martín de Porres }\end{array}$ & Perú & 6 & $10,17 \%$ \\
\hline Caderno Virtual de Turismo & $\begin{array}{c}\text { Universidade Federal } \\
\text { do Rio de Janeiro }\end{array}$ & Brasil & 5 & $8,47 \%$ \\
\hline $\begin{array}{l}\text { RBTUR Revista Brasileira de } \\
\text { Pesquisa em Turismo }\end{array}$ & $\begin{array}{c}\text { Associação Nacional } \\
\text { de Pesquisa e Pós- } \\
\text { Graduação em } \\
\text { Turismo (ANPTUR) }\end{array}$ & Brasil & 5 & $8,47 \%$ \\
\hline Cuadernos de Turismo & Universidad de Murcia & España & 4 & $6,78 \%$ \\
\hline El Periplo Sustentable & $\begin{array}{c}\text { Universidad } \\
\text { Autónoma del Estado } \\
\text { de México }\end{array}$ & México & 3 & $5,08 \%$ \\
\hline Turismo y Sociedad & $\begin{array}{l}\text { Universidad } \\
\text { Externado de } \\
\text { Colombia }\end{array}$ & Colombia & 3 & $5,08 \%$ \\
\hline Investigaciones Turísticas & $\begin{array}{l}\text { Universidad de } \\
\text { Alicante }\end{array}$ & España & 2 & $3,39 \%$ \\
\hline Turismo - Visão e Ação & $\begin{array}{l}\text { Universidade do Vale } \\
\text { do Itajaí }\end{array}$ & Brasil & 1 & $1,69 \%$ \\
\hline Tourism \& Management Studies & $\begin{array}{l}\text { Universidade do } \\
\text { Algarve }\end{array}$ & Portugal & 1 & $1,69 \%$ \\
\hline $\begin{array}{l}\text { European journal of tourism } \\
\text { hospitality and recreation }\end{array}$ & $\begin{array}{l}\text { Instituto Politécnico } \\
\text { de Leiria }\end{array}$ & Portugal & 1 & $1,69 \%$ \\
\hline
\end{tabular}

Elaboración propia 


\subsection{Publicaciones por años}

Se han identificado los años 2015, 2016 y 2018 como aquellos en los que se publicaron más artículos, 8 en cada uno. En 2012 publicaron 6 artículos. En los años 2002, 2003, 2005, 2006 y 2007 no se identificaron artículos sobre el tema, lo que llama la atención, debido a que son los años posteriores a la Convención del PCI de la UNESCO (UNESCO, 2003), hecho que debió generar mayor reacción de la academia al ponerse al $\mathrm{PCl}$ como un tema formal de discusión y cuidado.

De ese modo, el número más antiguo del conjunto de las revistas objeto de estudio data del año 1991 (Estudios y Perspectivas en Turismo). Sin embargo, los artículos más antiguos sobre $\mathrm{PCl}$ son de 2001: "De la deconstrucción del patrimonio gastronómico peruano" (Benavides de Rivero, 2001) y "Patrimonio gastronómico peruano: realidades y perspectivas para un programa nacional" (Zapata, 2001), ambos publicados en la revista Turismo y Patrimonio (Perú). Los más recientes, hasta finales de 2019, fueron "Turismo, patrimonio cultural y desarrollo local. Evaluación del potencial turístico de aldeas rurales en la provincia de Entre Ríos, Argentina" (Tosselli, 2019), "Os signos e símbolos do patrimônio nas ações do marketing, uma relação possível? Reflexões a partir das campanhas da Feira Nacional do Doce (Fenadoce) de Pelotas/RS, Brasil" (Berselli et al., 2019) y "El potencial del enoturismo o ruta/rutas del vino en La Rioja" (Cabello, 2019); artículos publicados en las revistas PASOS Revista de Turismo y Patrimonio Cultural, RBTUR Revista Brasileira de Pesquisa em Turismo y El Periplo Sustentable, respectivamente.

El crecimiento, como puede verse, de publicaciones referidas al tema de $\mathrm{PCl}$ en revistas de turismo iberoamericanas no ha sido constante. No se puede hablar de una tendencia marcada. Es más, después de los artículos publicados en 2001, en 2004 se publican apenas 3 y nuevamente se produce un vacío hasta 2008.

Los resultados muestran, hasta aquí, que los estudios/publicaciones sobre $\mathrm{PCl}$ en revistas iberoamericanas de turismo son aún incipientes, tal como ocurre, por ejemplo, en el caso de las publicaciones sobre el turismo de base comunitaria vinculado al patrimonio (Graciano \& Holanda, 2020), esta última publicada en la revista brasileña -y regional por lo demás- RBTUR Revista Brasileira de Pesquisa em Turismo. Podría, entonces, pensarse que las pocas publicaciones son resultado de la poca emergencia sistemática del $\mathrm{PCl}$ como objeto de estudio-publicación en Iberoamérica, o bien que los autores prefieren publicar en revistas de otras disciplinas o en las que no son de habla española ni portuguesa (no iberoamericanas), aunque esto es discutible si se considera el porcentaje de participación ínfimo de autores sudamericanos en algunas de las revistas globales más importantes de turismo ${ }^{2}$ (Svensson et al., 2009) que se publican en inglés.

\footnotetext{
${ }^{2}$ Sobre autores centroamericanos no se tiene datos y de autores mexicanos, españoles y portugués tampoco de manera específica; en cambio, de América del Norte y Europa sí. La mención se hace con fines comparativos amplios.
} 
Figura 2. Artículos científicos sobre relación $\mathrm{PCl}$ y turismo publicados en revistas iberoamericanas según año de publicación.

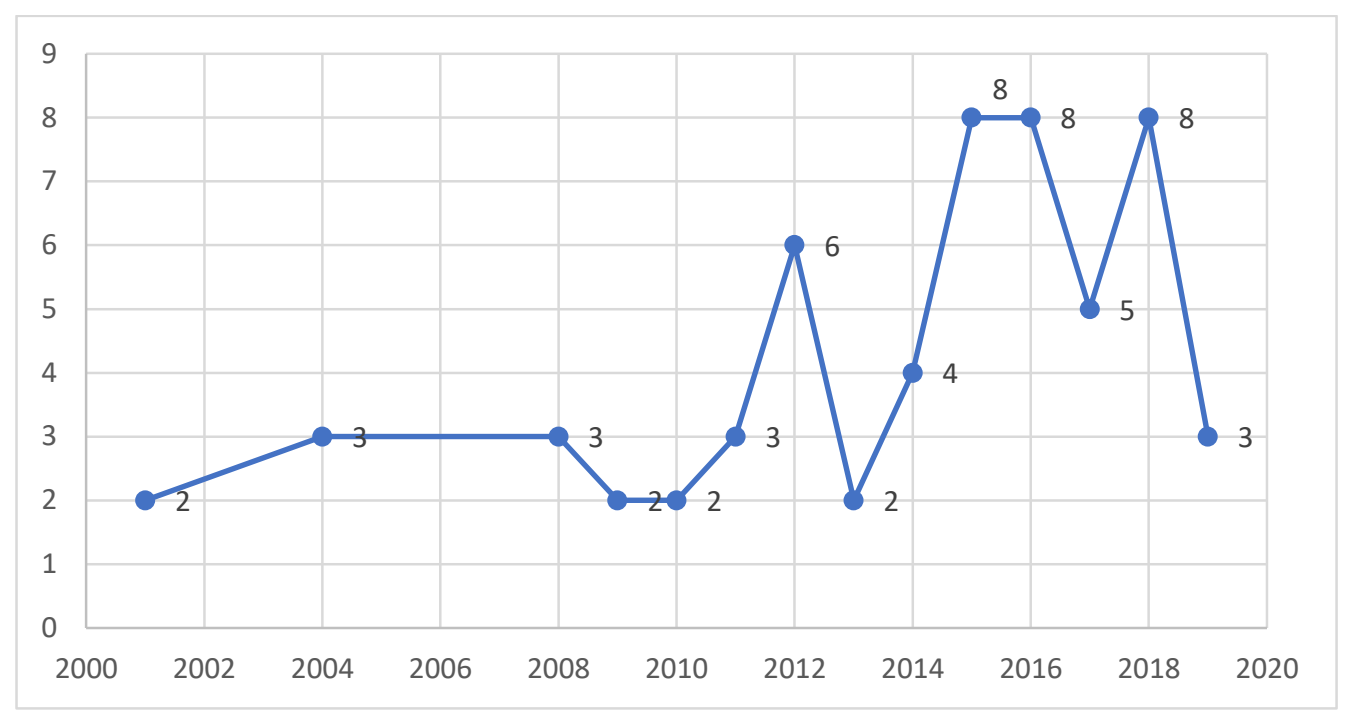

Elaboración propia

\subsection{Autores}

Los autores que han firmado los 59 artículos en estudio suman un total de 107. De estos el $62,62 \%$ son mujeres y el $37,38 \%$ hombres, diferente del caso de investigadores españoles, donde los autores más prolíficos son varones en un 75 \% (López-Bonilla et al., 2018) y de los tesistas doctorales también españoles, cuyas participaciones son equitativas, aunque con tendencia al crecimiento de la participación de mujeres (Peña et al., 2019).

Se indagó sobre los autores que han publicado más de una vez sobre el tema en cuestión sin importar la posición de aparición como coautor.

Los autores con filiación española de la Universidad de Córdoba Tomás López-Guzmán (López-Guzman et al., 2017; Moreno et al., 2016; Prada-Trigo et al., 2018) y Jesús Claudio Pérez Gálvez (López-Guzman et al., 2017; Moreno et al., 2016; Prada-Trigo et al., 2018) son quienes han publicado 3 artículos científicos cada uno y, en consecuencia, presentan un cierto interés por investigar y publicar trabajos que relacionan el $\mathrm{PCl}$ y turismo, además de haber colaborado entre ellos. Resaltan con 2 artículos - también con filiación española-, Elena Espeitx Bernat de la Universidad de Zaragoza (Espeitx, 2004; Rojas et al., 2016), Ivan Rêgo Aragão, cuyas filiaciones corresponden a los brasileños Instituto Federal de Sergipe y Universidad Estadual de Santa Cruz (Aragão, 2015; Aragão \& Ruiz de Macedo, 2011), Sandra Pesantez de los ecuatorianos Instituto Tecnológico Superior Luis Rogerio González y Universidad de Cuenca (López-Guzman et al., 2017; Prada-Trigo et al., 2018), José Prada-Trigo de la Universidad de Concepción de Chile (López-Guzman et al., 2017; Prada-Trigo et al., 2018) y Sergio Zapata Acha de la Universidad de San Martín de Porres del Perú (Zapata, 2001, 2009). Nótese que existe una gran concentración entre quienes más escriben sobre $\mathrm{PCl}$, pues de los 107 autores firmantes de los 59 artículos bajo análisis, solo 7 autores (el 6,54 \%) han publicado más de una vez.

En ese sentido, la mayoría de autores publicaron solo un artículo $(93,46 \%)$, lo que parece ser coherente con lo ocurrido en los casos del turismo de vinos (Durán-Sánchez et al., 2017), turismo comunitario basado en el patrimonio (Graciano \& Holanda, 2020), turismo 
religioso y peregrinación (Durán-Sánchez et al., 2018) y en investigaciones sobre patrimonio como factor de desarrollo del turismo (Álvarez-García, Maldonado-Erazo, Del Río-Rama, \& Castellano-Álvarez, 2019). Es decir, es identificable una marcada presencia de "autores transitorios" (Álvarez-García, Maldonado-Erazo, Del Río-Rama, \& Castellano-Álvarez, 2019) en desmedro de una minoría de especialistas con una larga carrera y mayor producción; poca especialización que también se da en las publicaciones sobre temas de gastronomía y comida (Okumus et al., 2018). La presencia de "pequeños productores" (Lotka, 1926, como se citó en Durán-Sánchez et al., 2018) en investigaciones relacionadas con el PCl es clara tanto en el caso de Iberoamérica como de otras realidades.

Tabla 2. Autores que han publicado dos o más artículos en las revistas seleccionadas de Iberoamérica.

\begin{tabular}{|c|c|c|c|}
\hline Autores & $\begin{array}{c}\text { Cantidad } \\
\text { artículos }\end{array}$ & Filiación & País \\
\hline López-Guzmán, Tomás & 3 & Universidad de Córdoba & España \\
\hline Pérez Gálvez, Jesús Claudio & 3 & Universidad de Córdoba & España \\
\hline Aragão, Ivan Rêgo & 2 & $\begin{array}{c}\text { Instituto Federal de Sergipe; Universidade } \\
\text { Estadual de Santa Cruz (UESC) }\end{array}$ & Brasil \\
\hline Espeitx Bernat, Elena & 2 & Universidad de Zaragoza & España \\
\hline Pesantez, Sandra & 2 & $\begin{array}{c}\text { Universidad de Cuenca; Instituto Tecnológico } \\
\text { Superior Luis Rogerio González }\end{array}$ & Ecuador \\
\hline Prada-Trigo, José & 2 & Universidad de Concepción & Chile \\
\hline Zapata Acha, Sergio & 2 & Universidad de San Martín de Porres & Perú \\
\hline
\end{tabular}

Elaboración propia

Sobre la cantidad de autores por artículo, la mayoría fueron firmados por un solo autor (24 equivalentes al 40,68 \%); los firmados por 2, 3 y 4 autores representan el 30,51 \%, 20,34 $\%$ y $8,47 \%$ respectivamente; en tanto que la media de coautoría es de 1,81 , muy por debajo del caso específico de la producción científica española en turismo en revistas de alto impacto y visibilidad 2,68 (López-Bonilla et al., 2018). El número máximo de autores por artículo fue 4, en 5 ocasiones. Desde otro ángulo, la mayoría de los artículos fueron escritos en colaboración $(59,32 \%)$, coincidiendo parcialmente con el caso de publicaciones sobre turismo de vinos en particular (Durán-Sánchez et al., 2017), aunque en ese estudio bibliométrico las colaboraciones alcanzaron las tres cuartas partes del total. En todo caso, se es consistente con la tendencia a desarrollar investigaciones en equipos (Hernández et al., 2014; Maletta, 2015; Niño, 2019).

Se tiene también que predominan las colaboraciones hechas por 2 y 3 investigadores, tal como ocurre en el caso de las colaboraciones en gastronomía y comida, identificadas en un estudio bibliométrico que analizó las 16 revistas líderes en los campos de turismo y hotelería (Okumus et al., 2018).

Siempre sobre las colaboraciones, los artículos que fueron firmados por más de un autor de la misma institución suman 20, mientras que los firmados por autores de diferentes filiaciones (colaboraciones interinstitucionales) son 15, y de estos, 6 por autores de países 
diferentes: Chile-España-Chile-España, España-Chile-España-Ecuador, Chile-España-EcuadorEspaña, México-México-España-México, Portugal-Brasil-Brasil, México-México-EspañaMéxico.

Las colaboraciones entre autores pueden ser locales (misma institución), domésticas (nacionales, pero de diferentes instituciones) e internacionales (de diferentes países) (Ardenuy, 2012, como se citó en López-Bonilla et al., 2018). En ese sentido, en la presente investigación son identificadas colaboraciones locales (20), domésticas (9) e internacionales (6). Del total, el 25,42\% de los artículos fue escrito, por lo tanto, por autores de diferentes procedencias, lo cual facilitó, por ende, el intercambio de puntos de vista y experiencias. Es decir, son limitadas las colaboraciones amplias o abiertas (domésticas e internacionales), pese a que en general las colaboraciones son señal de crecimiento de una disciplina, tendencia en la investigación actual (Maletta, 2015) y ofrecen "resultados provechosos para los países y/o destinos participantes" (Vidaurri, 2014, p. 37).

Las colaboraciones entre autores en investigaciones sobre comida y gastronomía en campos de hotelería y turismo han ido aumentando, y al igual que las de investigaciones sobre $\mathrm{PCl}$ en Iberoamérica, predominan las realizadas entre autores de la misma institución, lo que significa que las colaboraciones multidisciplinarias e internacionales deben aún promoverse (Okumus et al., 2018), específicamente en temas vinculados directa e indirectamente al PCl.

En tanto que los artículos firmados por 4 autores se han dado entre los años 2016, 2017 y 2018, es decir, en los últimos años, no obstante, en 2019 no se identificó ninguna colaboración así. Esta cantidad de autores colaborando es excepcional en el caso de los artículos en estudio; situación similar ocurre en el caso del análisis bibliométrico sobre las publicaciones en cocina y gastronomía (Okumus et al., 2018), donde un número mayor de 3 autores por artículo fue poco frecuente.

Tabla 3. Cantidad de autores por artículo

\begin{tabular}{|c|c|c|}
\hline Autores & Frecuencia & Porcentaje \\
\hline 1 & 24 & $40,68 \%$ \\
\hline 2 & 18 & $30,51 \%$ \\
\hline 3 & 12 & $20,34 \%$ \\
\hline 4 & 5 & $8,47 \%$ \\
\hline \multicolumn{3}{|c|}{ Elaboración propia }
\end{tabular}

\subsection{Filiaciones}

Al observar la cantidad de autores según el país de procedencia (por filiación), Brasil y España son los que sobresalen con 33 y 24 afiliados respectivamente, seguidos por Argentina y México con 13 y 11, Portugal y Perú con 11 y 5, Colombia y Chile con 4 y 3, y Ecuador y Reino Unido con 1 autor afiliados cada uno (ver figura 2), datos que permiten identificar una relación entre los autores más prolíficos y los países con mayor cantidad de autores (según su filiación).

A nivel de qué instituciones específicamente son las que acompañan las firmas de los autores (o filiaciones), resaltan las siguientes: Universidad Autónoma del Estado de México (México) en 7 firmas; las universidades de Córdoba (España), de San Martín de Porres (Perú) y Algarve (Portugal) en 6 oportunidades cada una; la Universidad de Girona (España) en 5 firmas, mientras que las universidades de Buenos Aires (Argentina), Lisboa (Portugal) y 
Estadual de Santa Cruz (Brasil), en 4 firmas cada una. Si se mira las instituciones con mayor cantidad de autores publicados en España, es decir, las universidades de las Islas Baleares, de Alicante, de Valencia, de Las Palmas de Gran Canarias, de Granada, Autónoma de Madrid, entre otras (López-Bonilla et al., 2018; López-Bonilla \& López-Bonilla, 2020), y las filiaciones de los autores más prolíficos internacionalmente, adscritos a la University of Surrey, Nanyang Technological University y la Universidad de Algarve (Strandberg et al., 2018), podrá verse que las universidades españolas más prolíficas no suelen publicar sobre el $\mathrm{PCl}$ en revistas iberoamericanas de turismo (entendible por sus indexaciones y ubicación en rankings), cosa que sí sucede excepcionalmente con la Universidad de Algarve (Portugal), la cual es la única identificada en esta investigación que figura entre las más prolíficas internacionalmente, además de entre las mejores 150 del ranking mundial de universidades (Shangai Ranking, 2020).

En cuanto a la cantidad de instituciones por país hay una notable diferencia entre el número de instituciones brasileñas y españolas (19 y 16 instituciones diferentes respectivamente) en relación al resto. En el caso de Argentina, se identificaron 8 instituciones, en México 5 y en Portugal 3. En los casos de Chile, Colombia y Ecuador 2 instituciones por país. $Y$ en el caso del Perú, solo una.

Pese a no ser concluyente, es posible sostener que hay una gran coincidencia entre los territorios que reciben más turistas y su producción científica (López-Bonilla et al., 2018; Peña et al., 2019; Vargas, 2014). Para el caso de la presente investigación, la relevancia de España, Brasil, Argentina, México y Portugal, en cuanto a cantidad de instituciones que hacen investigación y publican, se condice con la cantidad de llegadas internacionales, pues son esos mismos los países líderes (Organización Mundial del Turismo, 2019) si consideramos solo a los destinos-países de Iberoamérica. Si bien no es posible establecer una relación directa y firme entre el desarrollo turístico y la producción publicada en lberoamérica, sí es posible sugerirla con el fin de ahondar en el tema.

Figura 3. Porcentaje de autores según país de filiación

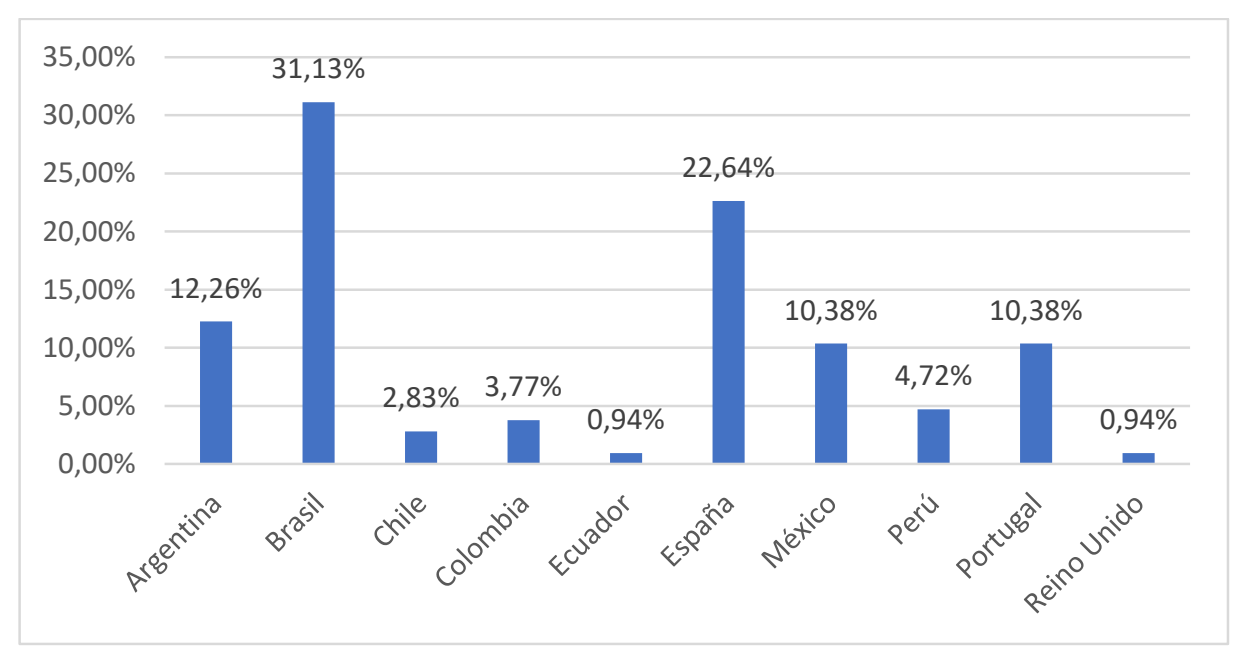

Elaboración propia 
Tabla 4. Instituciones con mayor cantidad de filiación según país.

\begin{tabular}{|c|c|c|}
\hline Institución & País & $\begin{array}{c}\text { Cantidad de } \\
\text { filiaciones }\end{array}$ \\
\hline Universidad Autónoma del Estado de México & México & 7 \\
\hline Universidad de Córdoba & España & 6 \\
\hline Universidad de San Martín de Porres & Perú & 6 \\
\hline Universidade do Algarve & Portugal & 6 \\
\hline Universidad de Girona & España & 5 \\
\hline Universidad de Buenos Aires & Argentina & 4 \\
\hline Universidade de Lisboa & Portugal & 4 \\
\hline Universidade Estadual de Santa Cruz & Brasil & 4 \\
\hline Fundación Universitaria Los Libertadores & Colombia & 3 \\
\hline Universidad de São Paulo & Brasil & 3 \\
\hline Universidad de Vigo & España & 3 \\
\hline Universidade Anhanguera - Uniderp & Brasil & 3 \\
\hline Universidade do Vale do Itajaí & Brasil & 3 \\
\hline Universidade de Caxias do Sul & Brasil & 2 \\
\hline Centro Universitário UNA & Brasil & 2 \\
\hline Instituto Federal de Sergipe & Brasil & 2 \\
\hline Universidad Autónoma de Chile & Chile & 2 \\
\hline Universidad de Concepción & Chile & 2 \\
\hline Universidad de Quintana Roo & México & 2 \\
\hline Universidad de Sevilla & España & 2 \\
\hline Universidad de Zaragoza & España & 2 \\
\hline Universidad Nacional de Mar del Plata & Argentina & 2 \\
\hline Universidad Nacional del Sur & Argentina & 2 \\
\hline Universidade Estadual de Ponta Grossa & Brasil & 2 \\
\hline Universidade Estadual do Centro Oeste - UNICENTRO & Brasil & 2 \\
\hline Universidad Feevale & Brasil & 2 \\
\hline
\end{tabular}

Elaboración propia

\subsection{Procedencia académica de autores}

Al indagar sobre la procedencia específica de los autores (según las filiaciones), es decir, orientación académica de la facultad, instituto, centro, departamento u otros de adscripción, se encontró que el 44,74 \% lo hizo desde espacios académicos vinculados al estudio directo del turismo, el 9,65 \% a Economía, el 7,89 \% a Antropología, el 7,02\% a Administración, Gestión y Empresas y el 7,02 \% a Geografía también; espacios académicos de procedencia relacionados con temas Ambientales fueron el 5,26\%, al Arte, Patrimonio y Museos 4,39 \%, a Sociología y Educación 2,63 \% cada una, y a Psicología y Nutrición 1,75\% cada una también.

Se tiene, al agrupar los resultados por disciplinas afines, que el 44,74\% procede de espacios académicos, específicamente de turismo con toda la amplitud y multidisciplinariedad, el 16,67 \% de las ciencias económicas y empresariales, el 14,91\% de las 
ciencias sociales, el $12,28 \%$ de las ciencias geográfico-ambientales y el 4,39 \% de las psicológica-educativas. Estos resultados difieren con los vinculados a los investigadores españoles en turismo y hostelería, donde si bien no aparece el turismo como espacio académico de procedencia de autores, dominan con claridad las ciencias económicas y empresariales ( $81 \%$ de los autores), seguido de espacios vinculados a la geografía, psicología y las ciencias sociales como antropología y sociología (López-Bonilla \& López-Bonilla, 2020). Es interesante ver, en todo caso, que en la región en estudio se viene consolidando el turismo como espacio de procedencia de investigación.

$Y$ al igual que en las investigaciones sobre patrimonio como medio de desarrollo local y los temas más importantes en turismo y hotelería en España (Álvarez-García, MaldonadoErazo, Del Río-Rama, \& Castellano-Álvarez, 2019; López-Bonilla \& López-Bonilla, 2020), las universidades son aquellos espacios donde en gran mayoría se genera el conocimiento publicado.

Figura 4. Origen disciplinar de autores (facultad, instituto, centro, departamento, otro, de procedencia)

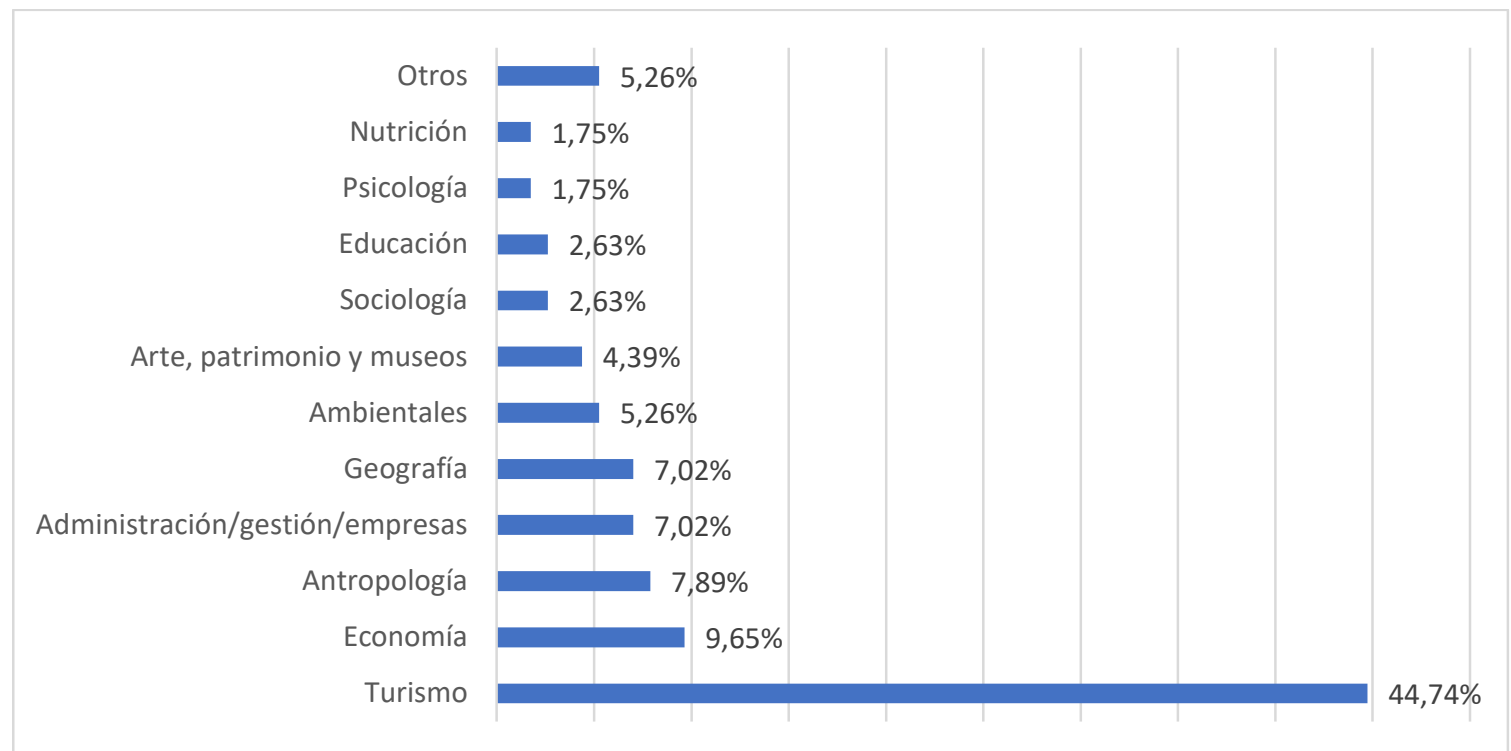

Elaboración propia

\subsection{Principales temáticas y enfoques}

Es importante resaltar que los artículos (96,62 \%) que forman parte de la investigación tienen como lugar de estudio realidades iberoamericanas, lo cual genera una mirada regional. En ese sentido, casi un tercio de los casos de estudio son de Brasil (32,20\%), seguido de España (13,56 \%), Argentina (11,86\%), Portugal (10,17\%), México y Perú (8,47 \% cada uno), Colombia (5,08\%), Ecuador y Chile (3,39 \% cada uno) y República Checa (1,69\%), mientras el 1,69 \% (1 artículo) no especifica el lugar de estudio. Es decir, salvo en un caso, en las publicaciones regionales analizadas, el foco está en las problemáticas y realidades regionales.

Ahora bien, en cuanto al tema general de cada artículo, se identificaron las siguientes categorías y resultados: el 25,42 \% se insertó dentro de "Patrimonio como recurso para el turismo", el 16,95 \% en "Educación patrimonial", el 16,95 \% en "Impactos en el patrimonio", el $13,56 \%$ en "Producto, marketing y patrimonio", el $11,86 \%$ en "Autenticidad del 
patrimonio", el 8,47 \% en "Tipologías del turismo asociadas al patrimonio" y el 6,78 \% en la categoría "Patrimonio y desarrollo". Lo que puede leerse es que hay un interés mayor por conocer las posibilidades de uso turístico del $\mathrm{PCl}$, es decir, se identifica el potencial del $\mathrm{PCl}$ como recurso (Garcia Canclini, 1999; Hernández, 2002).

Así también resaltan los temas de autenticidad e impactos en el patrimonio, que reunidos suman $28,81 \%$, lo cual podría reflejar la preocupación por las afectaciones causadas por el turismo. La educación patrimonial como tema también llama la atención por incorporar al turismo como medio de comunicación al ser una categoría vinculada al conocimiento, valoración y conservación del patrimonio.

Algunas de las principales temáticas identificadas, como "patrimonio como recurso para el turismo", "tipologías asociadas al turismo" y "los impactos en el patrimonio" (que suman 50,84\%), son coincidentes y consistentes con los temas: turismo cultural, las atracciones turísticas y los impactos del turismo, que son algunos de los 12 principales enfoques temáticos identificados en los estudios bibliométricos sobre turismo (Wickham, Dunn y Sweeney, 2012, como se citó en Koseoglu et al., 2016). Estos resultados, en cambio, no coinciden con algunas de las principales temáticas, según otros estudios vinculados a turismo: marketing, la gestión empresarial, economía, la geografía, etc. (García-Lillo et al., 2016; López-Bonilla \& López-Bonilla, 2020; Strandberg et al., 2018).

Sin embargo, desde la perspectiva de los estudios sobre PCI (Su et al., 2019), el enfoque estuvo en temas como el patrimonio mismo, espacios patrimoniales, protección del patrimonio, entre otros, lo cual generó una pequeña cantidad de estudios que trataron sobre el patrimonio y el desarrollo sostenible, donde podrían insertarse los estudios relacionados con el uso turístico. Es decir, la valoración del vínculo entre el turismo y del PCl varía según el sector que la estudia.

Respecto de los enfoques metodológicos de investigación, el 83,05\%, es decir, 49 de los 59 artículos, utilizó metodologías cualitativas; el 8,47\%, cuantitativas (5 artículos) y el 8,77 $\%$ metodologías mixtas (5 artículos). Cabe recordar que en la investigación solo se incluyeron artículos empíricos y no los de corte teórico. Es notorio el mayor uso de metodologías cualitativas, tal como ocurre con los casos de investigaciones sobre patrimonio como base de desarrollo turístico local (Álvarez-García, Maldonado-Erazo, Del Río-Rama, \& CastellanoÁlvarez, 2019; Graciano \& Holanda, 2020). Sin embargo, en el caso de los estudios sobre gastronomía y comida dominan los estudios de corte cuantitativo (Okumus et al., 2018). La prevalencia del uso de metodologías cualitativas puede entenderse como señal de que un tema (como es el caso del patrimonio como medio para desarrollo local) está aún en crecimiento (Álvarez-García, Maldonado-Erazo, Del Río-Rama, \& Castellano-Álvarez, 2019) y tiene un carácter más descriptivo, lo que es también consistente con la relativa nueva emergencia del turismo patrimonial (Espeso-Molinero, 2019; Timothy, 2018; World Tourism Organization, 2018, 2020).

\subsection{Co-ocurrencia de palabras en palabras clave y resúmenes}

Respecto del estudio, las palabras clave con mayor co-ocurrencia fueron "turismo", "patrimonio cultural", "turismo cultural", "patrimonio", y en menor medida "patrimonio cultural inmaterial", "gastronomía" y "desarrollo local" (ver figura 5). El primer grupo de palabras responde a la naturaleza propia de los temas de los artículos seleccionados, es decir, 
ser artículos que tratan temas de $\mathrm{PCl}$ en revistas de turismo y ser el uso turístico del $\mathrm{PCl}$ en la forma de turismo cultural una de las principales formas de "consumo", además de tendencia (Espeso-Molinero, 2019; Martorell, 2017; Richards, 2018a; Timothy, 2018; World Tourism Organization, 2018, 2020). Así también, las palabras clave están funcionando como descriptores, pues representan el contenido del artículo identificado en las principales temáticas (ver punto 3.6). Cabe señalar que en el caso de las palabras "patrimonio cultural inmaterial", en tanto, uno de los principales términos identificados, su uso no es mayor debido a la dispersión al utilizarse los términos "patrimonio intangible" o "patrimonio inmaterial", como puede verse en la figura 5. Los resultados, por cierto, permiten confirmar el cumplimiento de la ley de Zipf, relacionada con la frecuencia de palabras, donde una pequeña cantidad de estas se repite muchas veces (Quevedo-Silva et al., 2016).

Puede verse, asimismo, la subrepresentación de los textos de algunos artículos, pues términos sin co-ocurrencias o que tienen co-ocurrencias como "tango", "Brasil", "mercantilización" o "políticas públicas", no funcionan como descriptores del contenido, perdiéndose así la oportunidad de ser identificables, idea central del uso de palabras clave en textos. La subrepresentación parece indicar, por cierto, la presencia de autores que designan palabras clave solo como parte de un trámite editorial (Macías-Alegre, 2016) y con propósitos de identificación. 
Figura 5. Mapa de densidad de mínimo 2 co-ocurrencia de palabras clave.

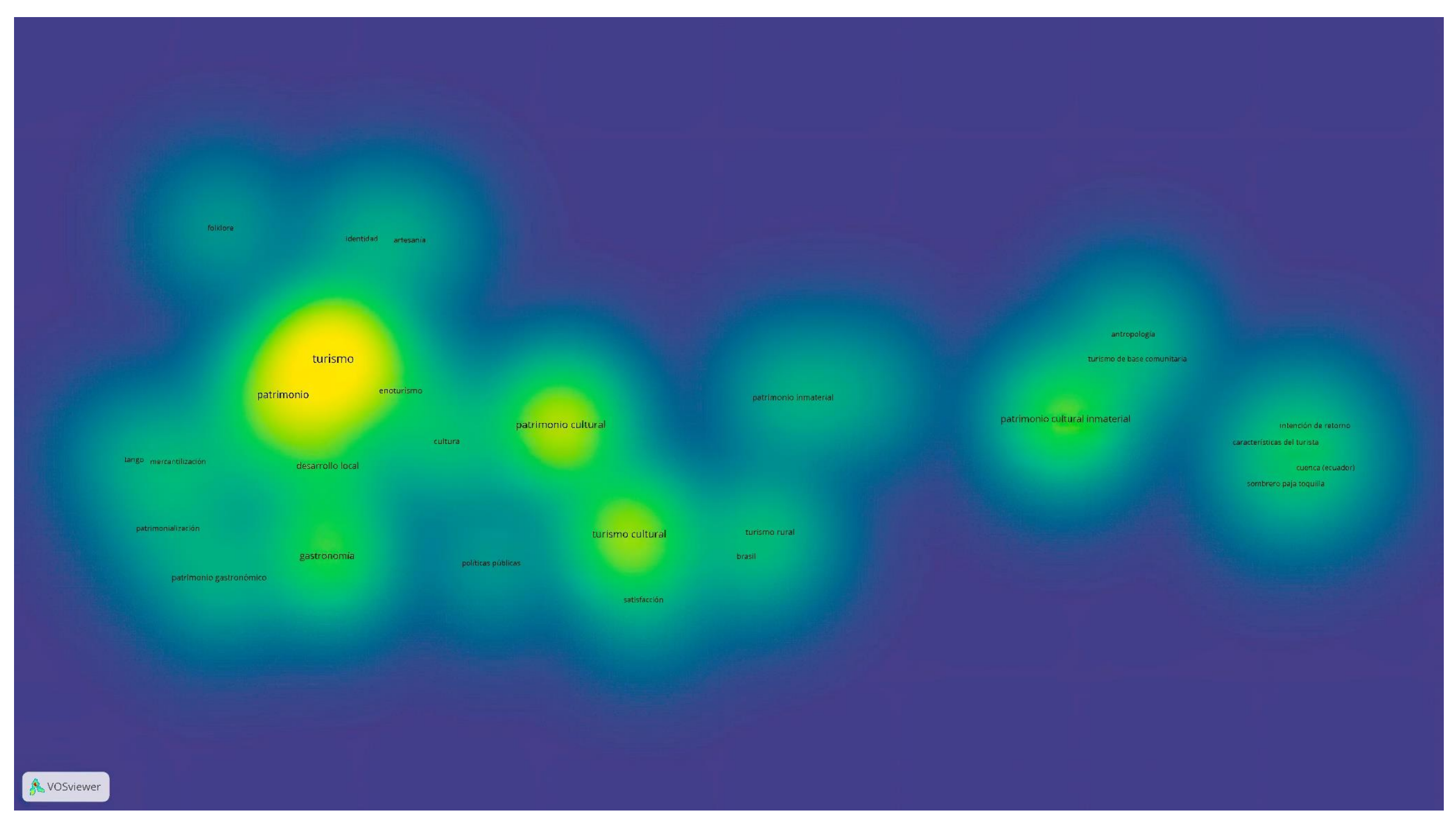

Elaboración propia 
Figura 6. Mapa de densidad de mínimo 5 co-ocurrencia de palabras clave.

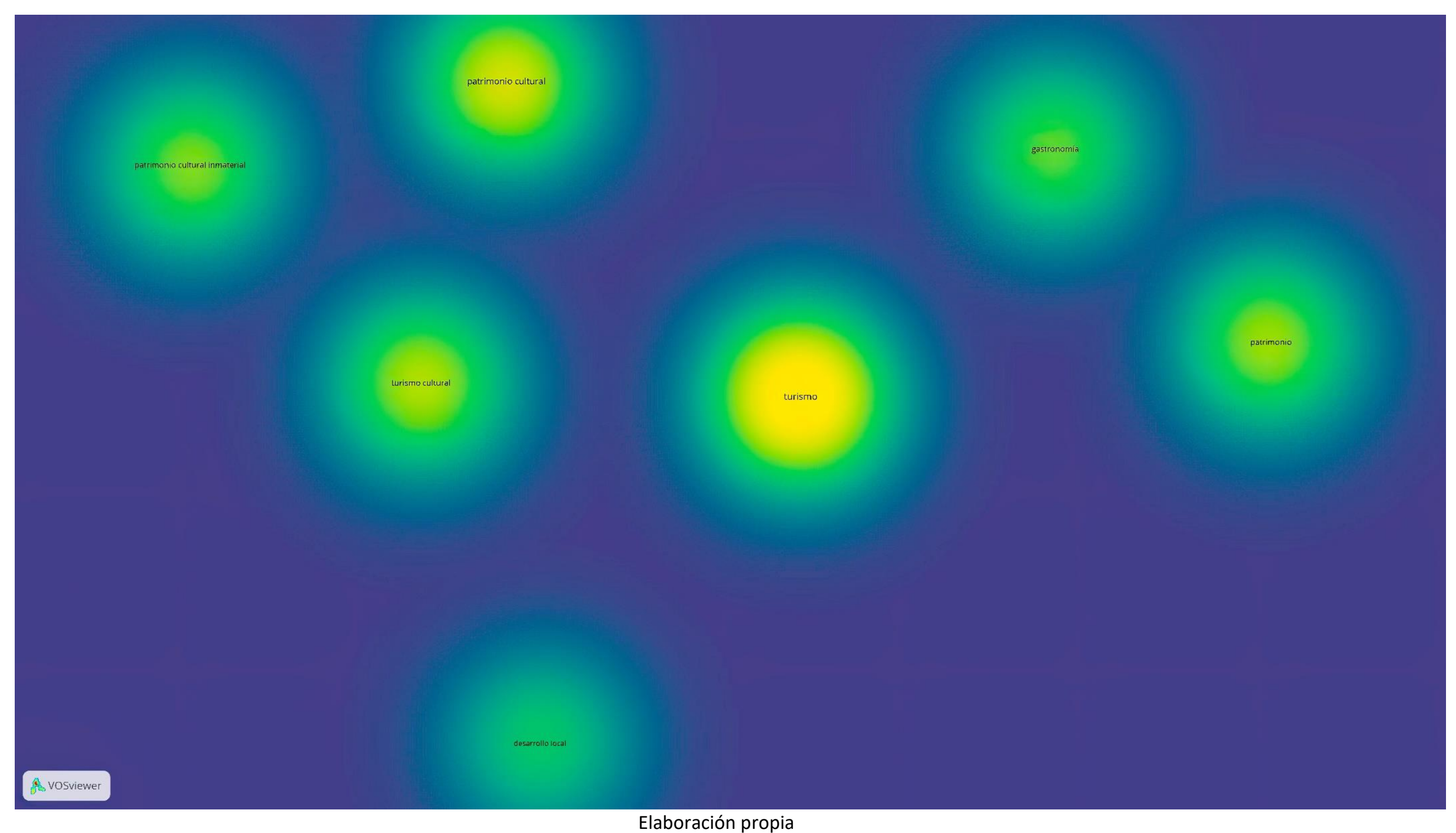


Figura 7. Mapa de relaciones entre palabras clave con mínimo 5 co-ocurrencias.

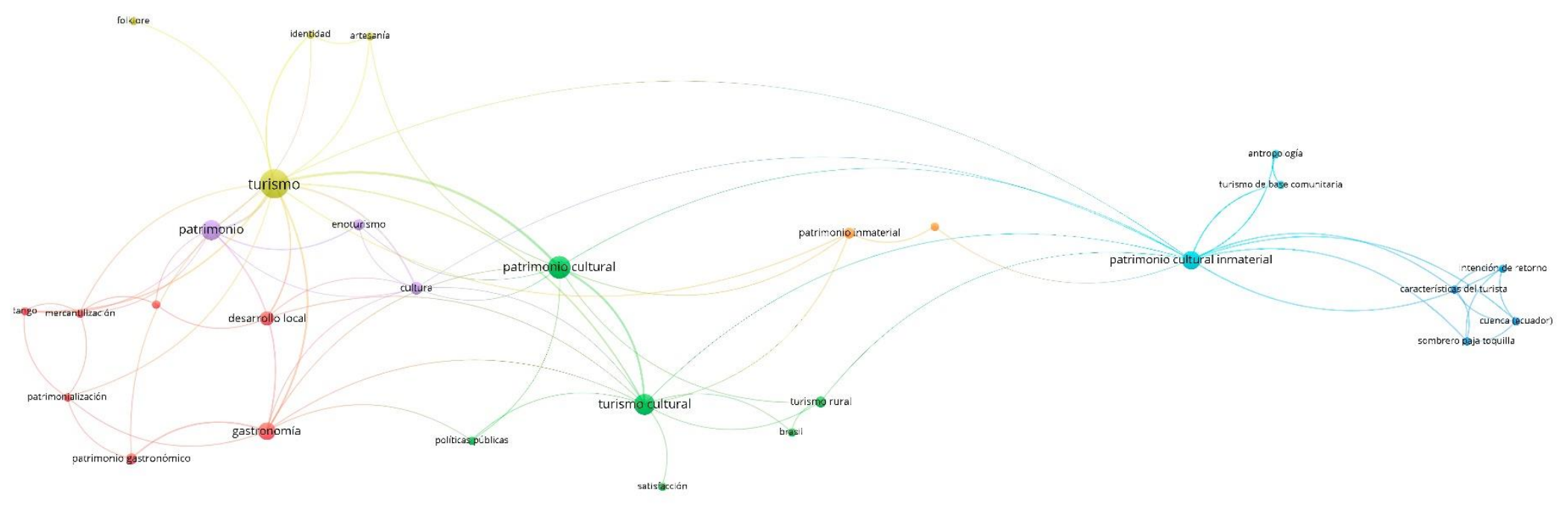


Las conexiones entre términos, de manera amplia, reflejan la vinculación entre el turismo, patrimonio cultural y el $\mathrm{PCl}$, lo que refleja el uso turístico del patrimonio, especialmente en la forma de turismo cultural. Lo que llama la atención por su relevancia y transversalidad es que el término "sostenibilidad" no aparezca en las conexiones.

Si se mira las relaciones entre los principales términos con co-ocurrencias, se tiene que "patrimonio cultural" se relaciona con "turismo cultural", a razón del uso que el primero hace del segundo; que el término "turismo" tiene conexiones con el término "identidad", lo que se entendería por la naturaleza de lo que se oferta: un patrimonio vivo y muy característico de cada comunidad; que el término "desarrollo local" se relacione con "gastronomía" indicaría que esta manifestación del $\mathrm{PCl}$ es la dominante en el turismo cultural basado en el PCl y que su enfoque está en la búsqueda de desarrollo.

Por otro lado, en la figura 7 puede evidenciarse, una vez más, la dispersión en el uso de términos como "patrimonio" y "patrimonio cultural", y "patrimonio inmaterial" y "patrimonio cultural inmaterial" (la dispersión alcanza al patrimonio intangible y patrimonio cultural intangible).

Por último, las manifestaciones del PCl que aparecen en el mapa (figura 7) vinculadas a los términos de mayor co-ocurrencia son "gastronomía", "folklore", "artesanía", "enoturismo", "turismo rural" y "turismo basado en la comunidad" (en ambos casos, generalmente por turismo basado en sus conocimientos), los cuales son, entonces, los aspectos del PCl identificados más sobresalientes en las investigaciones sobre su uso turístico.

\section{IV.CONCLUSIONES}

En la presente investigación se ha podido caracterizar la producción científica sobre el patrimonio cultural inmaterial publicada en revistas iberoamericanas de turismo, lo que es novedoso, debido a que no se identificaron estudios bibliométricos que analicen el tema de manera amplia o general, aunque sí se identificaron investigaciones respecto de manifestaciones específicas del PCl (gastronomía, vinos, religión).

La reducida cantidad de artículos sobre el tema (1,20\%) muestra un desfase entre las publicaciones y sus temáticas con respecto al turismo cultural patrimonial de corte experiencial, basado en el patrimonio - que es tendencia. Es decir, será labor de editores y autores profundizar en el tema y publicar más. En ese sentido, dado el reducido número de autores que publicaron más de una vez, se cree necesario promover la investigación en el tema, así como los espacios para sus publicaciones; también que se incorporen investigaciones básicas, aplicadas y el análisis de otras realidades, lo que enriquecería la producción científica de la región. Para tal fin, las colaboraciones internacionales y la promoción y ayudas para publicaciones serán indispensables.

Asimismo, las colaboraciones entre autores reflejan una vez más el localismo y regionalismo. Aún más, las colaboraciones internacionales fueron entre autores de los mismos países iberoamericanos, lo que puede entenderse por el idioma de publicación de los artículos (91,53 \% entre castellano y portugués). Sin embargo, más que una limitación puede ser una oportunidad para los editores y las revistas para incorporar con mayor decisión el inglés como idioma de publicación, como sucede por ejemplo con algunas de las siguientes importantes revistas de turismo de países no angloparlantes: Tourism and Hospitality Management (Croacia), European Journal of Futures Research (Alemania), European Journal of Tourism 
Research (Bulgaria), Sport Management Review (Países Bajos) (Scimago Institutions Rank, 2020) o algunas de las incluidas en esta investigación. Esto permitiría mayor alcance para las revistas, autores e instituciones.

Por otro lado, es posible afirmar, sin ser concluyentes, que existe una relación entre los destinos con mayor cantidad de visitantes por turismo y su producción y publicación en Iberoamérica, tal como ocurre, de la misma forma, en las principales potencias de turismo a nivel mundial (Vargas, 2014) y en el caso específico de España (López-Bonilla et al., 2018; Peña et al., 2019).

Así, el hecho de que la mayoría de los autores procedan de espacios académicos vinculados al turismo manifiesta su desarrollo como tema y espacio de generación de conocimiento en la región. Del mismo modo, cabe destacar la gran cantidad de mujeres como autoras publicadas $(62,62 \%)$, lo cual revela las oportunidades de las que disponen y su interés por el tema.

De igual manera, llama la atención que en el presente estudio no se identificaran claramente como categorías o temas de estudio la sostenibilidad, la aplicación de tecnologías en la comunicación e interpretación del $\mathrm{PCl}$ para los turistas o innovación en productos turísticos patrimoniales.

Por otra parte, las palabras clave con mayor co-ocurrencia y sus redes se relacionan con las temáticas identificadas en los artículos, es decir, los términos en líneas generales están funcionando como estructuras conceptuales y descriptoras de los artículos de investigación. Sin embargo, también es posible encontrar términos como "políticas públicas", "Brasil" o "mercantilización" y muchos otros que por su especificidad o ambigüedad no cumplen la función de facilitar la identificación del texto. Por este motivo, se recomienda que sean los editores de las publicaciones los encargados de determinar las palabras clave de los artículos (Macías-Alegre, 2016).

De este modo, el estudio del $\mathrm{PCl}$ en relación con el turismo en Iberoamérica debería ganar relevancia, ya que estos países cuentan con una amplia oferta turística cultural patrimonial, por lo que generar conocimiento y evaluar nuevas posibilidades de uso o consumo turístico más sostenible será fundamental. Esto se ha hecho más visible a raíz de la pandemia generada por la covid-19 que obligó a suspender el turismo por meses y, por ende, las posibilidades de uso y beneficios. Por lo tanto, urge indagar un modelo de turismo basado en el PCI más sostenible.

Finalmente, son limitaciones del estudio el excluir documentos como tesis, conferencias, libros, actas, informes y artículos de opinión, y hacer un uso limitado de los términos de búsqueda, toda vez que algunos autores pueden no establecer palabras clave que ayuden en la identificación del texto.

\section{REFERENCIAS BIBLIOGRÁFICAS}

Alberca, F. (2019). El turismo creativo. Conceptualización y características. Cultura(33), 145159.

Álvarez-García, J., Maldonado-Erazo, C., Del Río-Rama, M. d., \& Sánchez-Fernández, M. D. (2019). Creative tourism in small cities and rural areas: A bibliographic review. Enlightening Tourism. A Pathmaking Journal, 9(1), 63-94. 
Álvarez-García, J., Maldonado-Erazo, C. P., Del Río-Rama, M. d., \& Castellano-Álvarez, F. J. (2019). Cultural heritage and tourism basis for regional development: mapping of scientific coverage. Sustainability (Switzerland), 11(21), 1-21. doi: $10.3390 /$ su11216034

Aragão, l., \& Ruiz de Macedo, J. (2011). Turismo religioso, patrimônio e festa : Nosso Senhor dos Passos na cidade sergipana de São Cristóvão. Caderno Virtual de Turismo, 11(3), 399-414. Recuperado de http://www.ivt.coppe.ufri.br/caderno/index.php/caderno/article/view/611

Aragão, I. R. (2015). Turismo étnico e cultural: a coroação da rainha das taieiras como atrativo turístico potencial em Laranjeiras (SE). Caderno Virtual de Turismo, 15(2), 195-210. Recuperado http://www.ivt.coppe.ufri.br/caderno/index.php/caderno/article/view/1002

Benavides de Rivero, G. (2001). De la construcción del patrimonio gastronómico. Turismo y Patrimonio(3), 27-48. doi: 10.24265/turpatrim.2001.n3.02

Berselli, C., Torres Tricárico, L., \& Rossini, Diva de Mello. (2019). Os signos e símbolos do patrimônio nas ações do marketing, uma relação possível? Reflexões a partir das campanhas da Feira Nacional do Doce (Fenadoce) de Pelotas/RS, Brasil. 13(1), 72-91. doi: 10.7784/rbtur.v13i1.1479

Cabello, S. A. (2019). El potencial del enoturismo o ruta/rutas del vino en La Rioja. El Periplo Sustentable(37), 7-28. doi: 10.36677/elperiplo.v0i37.9116

Comerio, N., \& Strozzi, F. (2019). Tourism and its economic impact: A literature review using bibliometric tools. Tourism Economics, 25(1), 109-131. doi: $10.1177 / 1354816618793762$

Cominelli, F., \& Greffe, X. (2012). Intangible cultural heritage: Safeguarding for creativity. City, Culture and Society, 3(4), 245-250. doi: 10.1016/j.ccs.2012.10.003

Corral Marfil, J. A., \& Cànoves Valiente, G. (2013). La investigación turística publicada en revistas turísticas y no turísticas: análisis bibliométrico de la producción de las universidades catalanas. Cuadernos de Turismo(31), 55-81. Recuperado de https://revistas.um.es/turismo/article/view/170741

Durán-Sánchez, A., Álvarez-García, J., \& De la Cruz Del Río-Rama, M. (2018). Religious tourism and pilgrimage: bibliometric overview. Religions, 9(9), 1-15. doi: 10.3390/rel9090249

Durán-Sánchez, A., De la Cruz Del Río-Rama, M., \& Álvarez-García, J. (2017). Bibliometric analysis of publications on wine tourism in the databases Scopus and WoS. European Research on Management and Business Economics, 23(1), 8-15. doi: 10.1016/j.iedeen.2016.02.001

Ellis, A., Park, E., Kim, S., \& Yeoman, I. (2018). What is food tourism? Tourism Management, 68, 250-263. doi: 10.1016/j.tourman.2018.03.025

Espeso-Molinero, P. (2019). Tendencias del turismo cultural. PASOS Revista de Turismo y Patrimonio Cultural, 17(6), 1101-1112. doi: 10.25145/j.pasos.2019.17.076

Espeitx, E. (2004). Patrimonio alimentario y turismo: una relación singular. PASOS Revista de Turismo y Patrimonio Cultural, 2(2), 193-213. doi: 10.25145/j.pasos.2004.02.016 
Figueroa-Domecqa, C., Pritchard, A., Segovia-Péreza, M., Morgan, N., \& Villacé-Molinero, T. (2015). Tourism gender research: A critical accounting. Annals of Tourism Research, 52, 87-103. doi: 10.1016/j.annals.2015.02.001

Galvagno, M., \& Giaccone, S. C. (2019). Mapping Creative tourism research: Reviewing the field and outlining future directions. Journal of Hospitality \& Tourism Research, 43(8), 1256-1280. doi: 10.1177/1096348019862030

Gálvez, C. (2018). El campo de investigación del Análisis de Redes Sociales en el área de las Ciencias de la Documentación: un análisis de co-citación y co-palabras. Revista General de Información y Documentación, 28(2), 455-475. doi: 10.5209/RGID.62834

García Canclini, N. (1999). Los usos culturales del patrimonio cultural. En E. Aguilar Criado (Ed.), Patrimonio Etnológico. Nuevas perspectivas de estudio (págs. 16-33). Junta de Andalucía, Instituto Andaluz del Patrimonio Histórico. Recuperado de http://observatoriocultural.udgvirtual.udg.mx/repositorio/handle/123456789/130

García-Lilo, F., Úbeda-García, M., \& Marco Lajara, B. (2016). The intellectual structure of research in hospitality management: A literature review using bibliometric methods of the journal International Journal of Hospitality Management. International Journal of Hospitality Management, 52, 121-130. doi: 10.1016/j.ijhm.2015.10.007

Graciano, P. F., \& Holanda, L. A. (2020). Análise bibliométrica da produção científica sobre turismo de base comunitária de 2013 a 2018. Revista Brasileira de Pesquisa em Turismo, 14(1), 161-179. doi: 10.7784/rbtur.v14i1.1736

Hernández, F. (2002). El patrimonio cultural: la memoria recuperada. Gijón, Asturias: Ediciones Trea.

Hernández Sampieri, R., Fernández Collado, C., \& Baptista Lucio, M. d. (2014). Metodlogía de la investigación (6a ed.). McGRAW-HILL.

Koc, E., \& Boz, H. (2014). Triangulation in tourism research: A bibliometric study of top three tourism journals. Tourism Management Perspectives, 12, 9-14. doi: 10.1016/j.tmp.2014.06.003

Koseoglu, M. A., Rahimi, R., Okumus, F., \& Liu, J. (2016). Bibliometric studies in tourism. Annals of Tourism Research, 180-198. doi: 10.1016/j.annals.2016.10.006

López-Bonilla, J. M., Granados-Perea, C., \& López-Bonilla, L. (2018). Producción científica española en turismo: Un análisis de autoría basado en revistas internacionales con alto impacto y visibilidad. Cuadernos de Turismo(41), 343-367. doi: 10.6018/turismo.41.327061

López-Bonilla, J., \& López-Bonilla, L. M. (2020). Leading disciplines in tourism and hospitality research: a bibliometric analysis in Spain. Current Issues in Tourism, 1-17. doi: $10.1080 / 13683500.2020 .1760221$

López-Guzmán, T., Prada-Trigo, J., Pérez-Gálvez, J. C., \& Pesantez, S. (2017). El patrimonio inmaterial de la humanidad como herramienta de promoción de un destino turístico. Estudios y Perspectivas en Turismo, 26(3), 568-584. Recuperado de http://www.estudiosenturismo.com.ar/PDF/V26/N03/v26n3a04.pdf 
Macías-Alegre, A. (2016). ¿Tienen sentido las palabras clave de los artículos científicos? COMeIN. Revista de Currículum y Formación del Profesorado(55). Recuperado de http://comein.uoc.edu/divulgacio/comein/es/numero55/articles/Article-AdrianMacias-Alegre.html

Maletta, H. (2015). Hacer ciencia. Teoría y práctica de la producción científica (1a ed.). Lima: Universidad del Pacífico.

Martorell Carreño, A. (2017). Turismo cultural. Reflexiones para un encuentro sostenible entre turismo y cultura. Lima: Universidad de San Martín de Porres.

Meneguel, C. R., Rubio, S., \& Mundet, L. (2019). Análisis bibliométrico de la investigación turística sobre la ciudad de Girona. Rosa dos Ventos Turismo e Hospitalidade, 11(3), 598-614. doi: 10.18226/21789061.v11i3p598

Molina, S. (2016). Turismo creativo. Investigaciones Turísticas, 6, 170-173.

Mora-Torres, V. M., Serrano Barquín, R. d., \& Osorio García, M. (2017). El vínculo turismoperegrinación. Un acercamiento desde la producción científica en inglés y en español. Estudios y Perspectivas en Turismo, 26(1), 86-106. Recuperado de https://www.estudiosenturismo.com.ar/PDF/V26/N01/v26n1a05\%20.pdf

Moreno, R., Pérez-Gaálvez, J. C., Oyarce Ortuya, F., \& López-Guzmán, T. (2016). Factores de interés de un destino patrimonio de la humanidad. El caso de Valparaíso - Chile. Estudios y Perspectivas en Turismo, 25(3), 360-374. Recuperado de https://www.estudiosenturismo.com.ar/PDF/V25/N03/v25n3a07.pdf

Mota, L., Teixeira, S., \& Gonçalves, F. (2018). Remarks from tourism planning and sustainable tourism. A bibliometris study. Enlightening Tourism. A Pathmaking Journal, 8(2), 99127. Recuperado de http://hdl.handle.net/10272/15831

Mulet-Forteza, C., Genovart-Balaguer, J., Mauleon-Mendez, E., \& Merigó, J. (2019). A bibliometric research in the tourism, leisure and hospitality fields. Journal of Business Research, 101, 819-827. doi: 10.1016/j.jbusres.2018.12.002

Muñoz de Escalona, F. (2017). ¿Es el conocimiento del turismo una ciencia? Y si no lo es, ¿puede serlo? Turismo y Sociedad, 20, 27-48. doi: 10.18601/01207555.n20.02

Niño, V. M. (2019). Metodología de la investigación. Diseño, ejecución e informe (2a ed.). Bogotá: Ediciones de la U.

Okumus, B., Koseoglu, M., \& Ma, F. (2018). Food and gastronomy research in tourism and hospitality: A bibliometric analysis. International Journal of Hospitality Management, 73, 64-74. doi: 10.1016/j.ijhm.2018.01.020

Olivera, A. (2011). Patrimonio inmaterial, recurso turístico y espíritu de los territorios. Cuadernos de Turismo, 27, 663-677. Recuperado de https://revistas.um.es/turismo/article/view/140151

Organización Mundial del Turismo. (2019). Panorama del turismo internacional, edición 2019. Madrid: OMT. doi: 10.18111/9789284421237

Peña, A., Jiménez, M., Ruiz, J., \& Pontón, T. (2019). La producción científica en el "Sector de Hostelería y Turismo": Un análisis bibliométrico de las tesis doctorales españolas en el periodo 1978-2018. Investigaciones Turísticas, 71-94. 
Prada-Trigo, J., López-Gúzman, T., Pesántez, S., \& Pérez-Gálvez, J. (2018). Turismo cultural, patrimonio inmaterial y elementos que inciden en la diferente satisfacción del visitante a partir del estudio del sombrero de paja toquilla. Cuadernos de Turismo(41), 555-573. doi: 10.6018/turismo.41.327151

Quevedo-Silva, F., Santos, E. B., Brandão, M. M., \& Vils, L. (2016). Estudo Bibliométrico: Orientações sobre sua Aplicação. Revista Brasileira de Marketing, 15(2), 246-262. doi: 10.5585/remark.v15i2.3274

Richards, G. (2018a). Cultural tourism: A review of recent research and trends. Journal of Hospitality and Tourism Management, 36, 12-21. doi: 10.1016/j.jhtm.2018.03.005

Richards, G. (2018b). Turismo, uma força motriz subestimada para a economia criativa. Revista Turismo Em Análise, 29(3), 387-395. doi: 10.11606/issn.1984-4867.v29i3p387-395

Rojas Rivas, E., Viesca González, F. C., Espeitx Bernat, E., \& Quintero Salazar, B. (2016). El maguey, el pulque y las pulquerías de Toluca, Estado de México, ¿patrimonio gastronómico turístico? PASOS Revista De Turismo Y Patrimonio Cultural, 14(5), 11991215. doi: 10.25145/j.pasos.2016.14.080

Roigé, X., Del Mármol, C., \& Guil, M. (2019). Los usos del patrimonio inmaterial en la promoción del turismo. El caso del Pirineo catalán. PASOS Revista de Turismo y Patrimonio Cultural, 17(6), 1113-1126. doi: 10.25145/j.pasos.2019.17.077

Ross, D., Saxena, G., Correia, F., \& Deutz, P. (2017). Archaeological tourism: A creative approach. Annals of Tourism Research, 67, 37-47. doi: 10.1016/j.annals.2017.08.001

Ruhanen, L., Weiler, B., Moyle, B. D., \& McLennan, C.-I. J. (2015). Trends and patterns in sustainable tourism research: A 25-year bibliometric analysis. Journal of Sustainable Tourism, 23(4), 517-535. doi: 10.1080/09669582.2014.978790

Santucci Oliveira, B., Torres Tricárico, L., Lisboa Sohn, A. P., \& Pontes, N. (2020). The culinary intangible cultural heritage of UNESCO: A review of journal articles in EBSCO platform. Journal of Culinary Science \& Technology, 18(2). doi: 10.1080/15428052.2018.1513882

SCImago . (2020). Scimago Journal \& Country Rank. Recuperado de Scimago Journal \& Country Rank:

https://www.scimagojr.com/journalrank.php?category=1409\&page=2\&total size=12 $\underline{3}$

Shangai Ranking. (2020). ShanghaiRanking's Global Ranking of Academic Subjects 2020 Hospitality \& Tourism Management. Recuperado de Academic Ranking of World Universities: $\quad$ http://www.shanghairanking.com/shanghairanking-subjectrankings/hospitality-tourism-management.html

Strandberg, C., Nath, A., Hemmatdar, H., \& Jahwash, M. (2018). Tourism research in the new millennium: A bibliometric review of literature in Tourism and Hospitality Research. Tourism and Hospitality Research, 18(3), 269-285. doi: 10.1177/1467358416642010

Su, X., Li, X., \& Kang, Y. (2019). A bibliometric analysis of research on intangible cultural heritage using CiteSpace. SAGE Open, 9(2). doi: 10.1177/2158244019840119 
Tan, S.-K., Kung, S.-F., \& Luh, D.-B. (2013). A model of 'creative experience' in creative tourism. Annals of Tourism Research, 41, 153-174. doi: 10.1016/j.annals.2012.12.002

Timothy, D. J. (2018). Making sense of heritage tourism: Research trends in a maturing field of study. Tourism Management Perspectives, 25, 177-180. doi: 10.1016/j.tmp.2017.11.018

Toselli, C. (2019). Turismo, patrimonio cultural y desarrollo local. Evaluación del potencial turístico de aldeas rurales en la provincia de Entre Ríos, Argentina. PASOS Revista de Turismo y Patrimonio Cultural, 17(2), 343-361. doi: 10.25145/j.pasos.2019.17.024

UNESCO. (2003). Convención para la Salvaguardia del Patrimonio Cultural Inmaterial. Recuperado de UNESDOC. Biblioteca Digital: https://unesdoc.unesco.org/ark:/48223/pf0000132540 spa

UNESCO. (2008). World heritage information kit. Recuperado de UNESCO. World Heritage Centre: http://whc.unesco.org/uploads/activities/documents/activity-567-1.pdf

Van Eck, N. J., \& Waltman, L. (2010). Software survey: VOSviewer, a computer program for bibliometric mapping. Scientometrics, 84, 523-538. doi: 10.1007/s11192-009-0146-3

Vargas Sánchez, A. (2014). ¿Los principales destinos son también las principales potencias en la investigación en turismo?: la experiencia española. Turismo y Patrimonio(8), 15-30. doi: 10.24265/turpatrim.2014.n8.01

Vidaurri Arechiga, J. E. (2014). Atisbos a la investigación turística en México. Origen, evolución y situación actual. Turismo y Patrimonio(8). doi: 10.24265/turpatrim.2014.n8.02

World Tourism Organization. (2020). Tourism and culture. Recuperado de Organización Mundial de Turismo: https://www.unwto.org/es/tourism-and-culture

World Tourism Organization. (2018). Tourism and culture sinergies. Madrid: UNWTO. doi: $10.18111 / 9789284418978$

Zapata Acha, S. (2009). "Patrimonialización" de la gastronomía peruana. Turismo y Patrimonio(6), 17-28. doi: 10.24265/turpatrim.2009.n6.02

Zapata Acha, S. (2001). Patrimonio gastronómico peruano: realidades y perspectivas para un programa nacional. Turismo y Patrimonio(3), 9-24. doi: 10.24265/turpatrim.2001.n3.01

Župič, I., \& Čater, T. (2015). Bibliometric Methods in Management and Organization. Organizational Research Methods, 429-472. doi: 10.1177/1094428114562629

\section{CONTRIBUCIONES DE LOS AUTORES:}

Autor 1: Concepción del trabajo; análisis, adquisición e interpretación de los datos, redacción y revisión crítica del contenido, aprobación final de la versión a publicar.

Autor 2: Análisis, adquisición e interpretación de los datos; revisión crítica del contenido; aprobación final de la versión a publicar. 


\section{AGRADECIMIENTOS:}

El proyecto marco del presente artículo fue financiado por la Universidad de San Martín de Porres (USMP), Escuela Profesional de Turismo y Hotelería. Los autores desean agradecer a los revisores pares anónimos por sus importantes contribuciones para la mejora del artículo. Así también, agradecen el apoyo y recomendaciones del doctor en Ciencias de la Información por la Universidade de Brasília de Julio Santillán-Aldana y el apoyo de las estudiantes de la carrera de Turismo y Hotelería de la USMP Kathleen Chávez Recuay y Nicol Rufasto Ucañan. 